\title{
¿Hay alguna geografía humana que no sea geografía histórica? 1
}

\author{
Enric Mendizábal²
}

\begin{abstract}
RESUMEN
Las reflexiones teóricas sobre temas geográficos son fundamentales para el avance de la disciplina. En este texto se propone ver cómo el tiempo y la historia son elementos implícitos y no excesivamente explícitos en las investigaciones en geografía humana, así como la utilidad de tener presente la historicidad para hacer geografía. Se hace un repaso de algunos textos teóricos y metodológicos que se consideran importantes sobre cómo se ha analizado el tiempo desde la geografía humana, así como la propuesta de una tipología de conceptos temporales (cambio, evolución, transformación, proceso) que permite clasificar desde una nueva visión los trabajos empíricos sobre las distintas partes de la superficie de la Tierra.
\end{abstract}

Palabras clave: Geografía humana, geografía histórica, historia de la geografía, tiempo.

\begin{abstract}
The theoretical reflections on geographic subjects are fundamental for the advance of the discipline. I propose in this text how time and history are implicit elements and no excessively explicit in human geography research, as well as the utility to have present the historicity to do geography. There is a review of some theoretical and methodological texts that I consider relevant because they has analysed the time from the view of human geography, as well as it proposes a tipology of time concepts (change, evolution, transformation, process) that allows to classify from a new view the empirical works on the distinct parts of the surface of the Earth.
\end{abstract}

Key words: Human geography, historical geography, history of geography, time.

1 Este texto se ha elaborado en el marco de las discusiones teóricas realizadas con los miembros del Grup de Geografia Aplicada (2009SGR106), financiado por la Generalitat de Catalunya (2009-2013) y el proyecto de investigación Los paisajes de las áreas de montaña. Patrones de gestión de ocupación del territorio (CSO2009-08271) financiado por el Ministerio de Ciencia e Innovación de España (2009-2012). Agradezco los comentarios de Antoni Tulla, investigador principal y de las/los colegas Anna Badia, Albert Pèlachs, Joan Manuel Soria- no y Ana Vera, así como la inestimable ayuda de Ariadna García para el diseño y elaboración de las figuras. Asimismo, este texto le debe mucho a Abel Albet, compañero de despacho con quien comparto muchos más intereses que los estrictamente geográficos. Artículo recibido el 30 de enero de 2012, aceptado el 20 de mayo de 2012 y corregido el 8 de junio de 2012.

2 Departament de Geografia, Universitat Autònoma de Barcelona (España). E-mail: enric.mendizabal@uab.cat 
La geografía histórica no debería ser tanto una rama separada exclusiva y excluyente dentro de la geografía como una forma de realizar la investigación en geografía humana. Deberíamos saber que la simple descripción de la superficie terrestre es poco útil, y que para analizar y explicar paisajes, regiones, espacios, territorios $y / 0$ lugares (o cualquier otro nombre que demos a la parte de la superficie terrestre que estamos estudiando), estos deben ser interpretados como cambios, evoluciones, transformaciones y procesos temporales en la superficie terrestre, y este es el objetivo del siguiente texto. En el primer apartado se presenta la importancia de la variable tiempo en geografía recordando algunos textos de geografía clásicos, antiguos, modernos y actuales (y algunos de los anteriores posiblemente anticuados) para ver cómo se ha utilizado el tiempo en geografía humana. En el segundo apartado se muestran una serie de estudios geográficos, principalmente de lo que se puede considerar como geografía histórica en un sentido muy amplio de tal subdisciplina, ordenados a partir de una tipología de conceptos temporales que son sutilmente distintos (lo que en los estudios lingüísticos se conoce como "casi sinónimos"): cambio, evolución, transformación y proceso. En el tercer apartado hay unas reflexiones sobre la importancia que tiene poder hacer una geografía humana donde el tiempo y la historia estén presentes de manera implícita y explícita. Los estudios geográficos citados en la bibliografía corresponden a las lecturas arbitrarias del autor desde que empezó a estudiar geografía en la universidad en un lejano 1979.

\section{Si no hay tiempo (¿historia?), no hay geografía}

\section{¿Se puede definir qué es geografía humana?}

Efectivamente, se puede definir. Y cada definición tiene razones suficientes que la justifican. Así que no haré una investigación filológica para la definición de geografía, como tampoco haré un largo recorrido sistemático de las mil y una definiciones que existen de geografía. Sencillamente, elijo dos de similares que me sirven para orientar las reflexiones del presente texto, y que me han servido para orientar mi trabajo como geógrafo desde que las leí por primera vez. Son las de Peter Gould y Lluís Casassas, ambas casi idénticas: Peter Gould (1985: 116) escribe que "después de todo, la geografía humana trata de los seres humanos moviéndose (entre otras cosas) en el espacio geográfico". Para Lluís Casassas (1988: 43) "la geografía es la ciencia que estudia los movimientos de los hombres sobre la Tierra a lo largo del tiempo" ${ }^{\prime 3}$.

¿Por qué los movimientos de las personas sobre la Tierra? Zelinsky (1971) expuso un esquema evolutivo de los movimientos del ser humano sobre la Tierra al que da el nombre de transición de la movilidad. Este no es el lugar para explicar todo el esquema, y solamente haré referencia a la cuarta etapa de esta transición que Zelinsky caracteriza por una alta movilidad residencial, por un gran número de migraciones ciudad-ciudad, por una movilidad cotidiana por razones de trabajo muy considerable, así como por recibir inmigración extranjera. Todo esto debería de coincidir con una sociedad muy industrializada o postindustrial donde la transición demográfica haya terminado. Zelinsky da mucha importancia a la circulación habitual, ya que es uno de los elementos típicos de las zonas urbanizadas e industriales. En esta cuarta etapa, "más personas están haciendo viajes más largos por una gran diversidad de razones más a menudo que en cualquier momento anterior" (Zelinsky, 1971: 246). Todas estas ideas de Zelinsky muestran con claridad algunos de los temas básicos que estudia la geografía humana actual.

El párrafo anterior nos lleva a una segunda pregunta: ¿por qué los movimientos del ser humano a lo largo del tiempo? Porque el tiempo (la historia en sentido amplio) es una variable indispensable para hacer geografía; unos comentarios serán suficientes para mostrar esta idea.

\section{La maldición de Fred K. Schaefer}

Tampoco es necesario hacer un repaso de la historia de la geografía para tener presente

\footnotetext{
3 Sobre la obra del geógrafo de origen británico Peter Gould (1932-2000) puede consultarse Haggett (2003). Sobre la obra de Lluís Casassas (1922-1992), puede consultarse Mendizábal (1993b).
} 
la importancia del texto de 1953 de Fred K. Schaefer, Exceptionalism in Geography ${ }^{4}$, que supuso una ruptura fundamental en la manera de hacer geografía, y promovió los suficientes cambios para identificar a ese artículo como uno de los textos básicos y clásicos que toda persona interesada en ejercer de geógrafa debería leer y releer ${ }^{5}$. Pero para la geografía histórica, el texto de Schaefer fue una auténtica maldición. Horacio Capel tradujo el texto donde aparece esta cita:

"Hay un aspecto importante en el que la geografía difiere de las otras ciencias sociales. Estas últimas, al madurar, se concentran más y más en el descubrimiento de leyes de procesos, es decir, leyes que son en un aspecto importante como las leyes de la astronomía newtoniana. Dada la situación de un sistema en un cierto punto en el tiempo, las leyes de procesos permiten la predicción de los cambios que ocurrirán. La geografía es esencialmente morfológica. Las leyes estrictamente geográficas no contienen referencias al tiempo y al cambio. Con ello no pretendemos afirmar que las estructuras espaciales que exploramos no sean como cualquier estructura, el resultado de procesos. Pero el geógrafo, en su mayor parte, trata de esas estructuras tal como las encuentra, es decir, ya elaboradas" (Schaefer, 1977: 73).

La nueva geografía teorético-cuantitativa supuso la implantación en el mundo anglosajón (y donde este influyó) de una manera de hacer que rompía con la tradición geográfica de la geografía regional posibilista relacionada con Paul Vidal de la Blache en Francia, Siegfried Passarge en Alemania y Carl Sauer en Estados Unidos de América (y los seguidores respectivos). Tampoco es aquí el lugar de repasar todas (o algunas de) las historias de la geografía y de las discusiones sobre esta "nueva geografía". Solamente hay que tener presente la dificultad de incorporar el tiempo y el cambio en los modelos cuantitativos.

\footnotetext{
4 Cito solamente tres textos que considero clásicos sobre el tema: el estudio introductorio de Capel (en Schaeffer, 1977), Johnston (1983: 50-93) y García Ramón (1985: 57-66).

5 Es uno de los 41 textos seleccionados en Agnew, Livingstone y Rogers (1996).
}

A pesar de la maldición sobre el estudio del cambio en geografía en el texto seminal de la nueva geografía teorético-cuantitativa, se hizo un esfuerzo para introducirlo. Así, el capítulo 21 del libro de David Harvey (1969) se titula "Tipos de explicación temporal en geografía", y en su primera parte empieza reflexionando sobre la importancia de los estudios genéticos en geografía: la geografía histórica y la geomorfología (derivada de W.M. Davis) se citan ampliamente.

Harvey (1969, capítulo 21) explica el "descubrimiento" del tiempo por los científicos, en cuanto que incluyen las nociones de desarrollo, evolución y cambio a lo largo del tiempo, ante el mundo estático de los mecanicistas como Newton o Descartes. En la segunda parte del capítulo, Harvey se dedica a las explicaciones temporales en geografía, que clasifica en tres grandes grupos. El primer grupo es la narrativa, que es una descripción de una sucesión de acontecimientos en términos cronológicos. El segundo considera al tiempo como una variable de identidad que actúa y que tiene efectos causales; el establecimiento de una secuencia de etapas significa que cualquier área tiene que pasar por ellas; los ejemplos dados son los de desarrollo económico de Rostow y el ciclo de erosión de Davis. El tercero lo forman las explicaciones mediante procesos reales; Harvey escribe que en geografía se tienen pocas luces de cuáles son realmente estos procesos.

\section{El tiempo, un concepto (¿poco?) explícito en geografía}

El tiempo, como indicador de cambio, evolución, transformación o proceso está más o menos presente en todas las ciencias sociales, aunque no siempre de una manera explícita. Y en el campo de la geografía, a pesar de Schaefer y de la geografía morfológica, el tiempo ha sido un elemento básico para el análisis geográfico.

La Figura $N^{0} 1$ permite ver la distribución aproximada de la cantidad de trabajos que se realizan en la disciplina "geografía humana" en relación al período de tiempo estudiado. Primera cuestión que se debe tener cuando realizamos una investigación en geografía: nunca podemos estudiar el ahora, ya que 
Figura $\mathrm{N}^{\circ} 1$

¿Qué y cuánto estudia la geografía en relación con el tiempo?

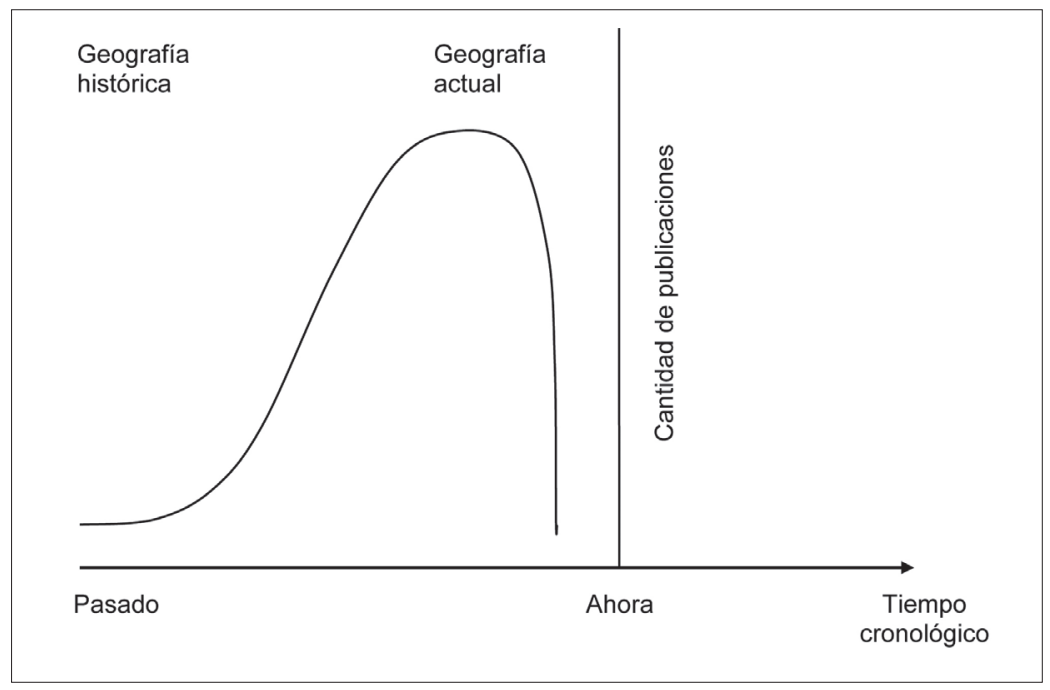

Fuente: adaptación de Enric Mendizábal y Ariadna García de la Figura 5.10 de Peter Haggett (1988: 620)

matemáticamente por tal concepto se entiende un instante infinitamente pequeño, de lo que se deduce que solamente podemos tener información del pasado y podemos imaginarnos el futuro. Si pensamos en un estudio cualesquiera de población, nunca podemos estudiar la población actual, ya que constantemente hay nacimientos, defunciones y migraciones $^{6}$. Así las cosas, argumentando hasta el límite, toda geografía es siempre geografía histórica, ya que los datos que se analizan pertenecen siempre al pasado.

\footnotetext{
6 La página web http://www.worldometers.info (última consulta: 28 de enero de 2012) ofrece la actualización instantánea de unos 50 datos distintos, entre los cuales los nacimientos, defunciones y crecimiento total de la población mundial. Es obvio que las cifras que ofrecen son criticables metodológicamente, pero la rapidez en que cambia la población, o las hectáreas deforestadas, o los barriles de petróleo llenados a lo largo del día en que se consulta esta base de datos, permite comprender la afirmación de la imposibilidad de estudiar el presente con exactitud. Las fotografías de los satélites que permiten las técnicas de la teledetección son siempre pasado: ¿hay que recordar las imágenes del terremoto de Japón de febrero de 2010 para comprender que nunca podemos analizar el ahora instantáneo?
}

Continuando con esta reflexión, la definición del ahora en un sentido más amplio, permite diferenciar la geografía actual de la geografía histórica. La Figura $N^{0} 2$ reproduce las reflexiones de Braudel (1958) sobre las escalas del tiempo que afectan al movimiento de las sociedades. La primera escala es el tiempo corto, que trata de los acontecimientos de la vida cotidiana del individuo y de la política: es el "ahora". La segunda escala es el tiempo medio, que trata de las oscilaciones y las coyunturas de la historia económica y social, caracterizadas por los ciclos económicos: es el "actualmente". La tercera escala es el tiempo largo, que trata de las estructuras, entendidas como la organización coherente de unas relaciones suficientemente fijas entre sociedad y territorio, y que muestran las permanencias: es el (largo) presente. La dificultad de diferenciar estas tres escalas de tiempo viene dada porque todas tres se deben medir en la misma escala cronológica que marca el tiempo astronómico y suceden a la vez en el mismo espacio, lugar, región, territorio y/o paisaje.

Uno de los elementos esenciales para el análisis geográfico es la escala espacial, ya que depende de qué escala se utilice, el tipo de análisis deberá variar, así como los con- 
Figura $\mathrm{N}^{\circ} 2$

Las distintas escalas del tiempo para explicar el ahora/el presente

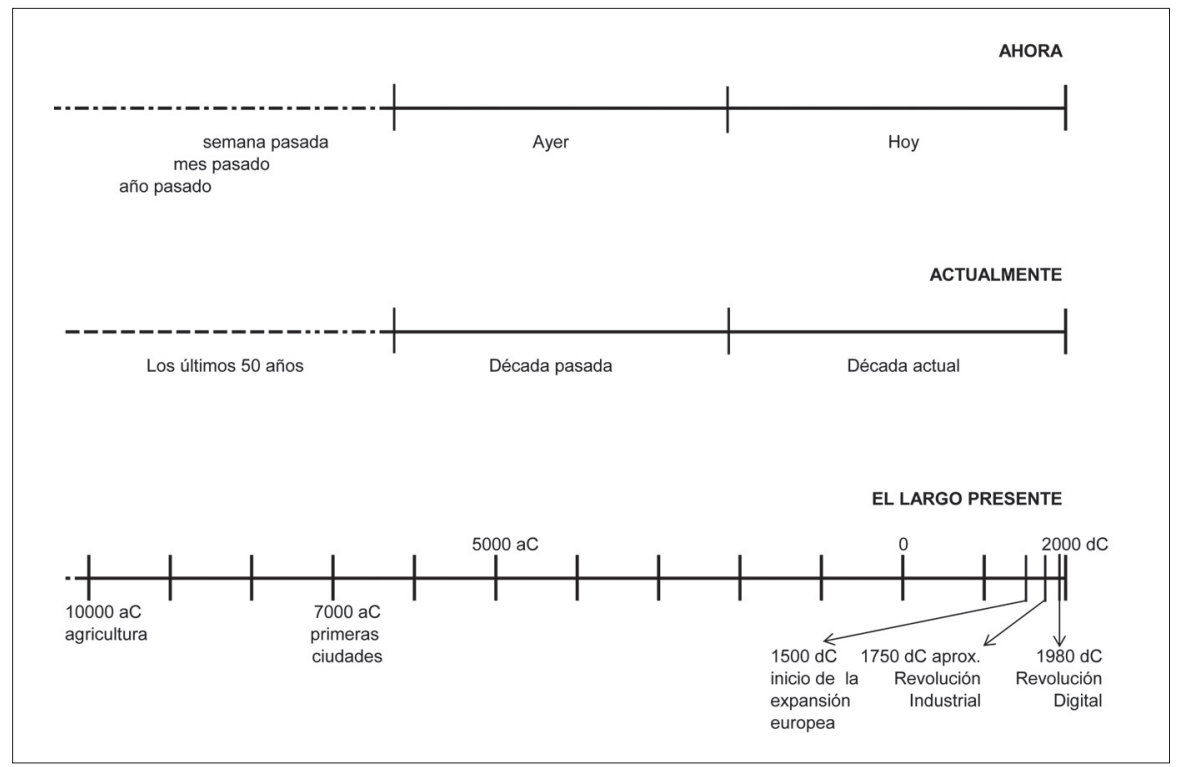

Fuente: adaptación de Enric Mendizábal y Ariadna García a partir de Rosenberg y Grafton (2009: 25).

ceptos utilizados: "un mismo geógrafo puede proceder al estudio de los problemas de una aldea africana, al análisis de la situación de la región donde se encuentra esta aldea, al examen de los problemas al nivel del Estado en que se inscribe y a la comprensión del 'subdesarrollo'al nivel del conjunto del 'tercer mundo'" (Lacoste, 1977: 56). En la Figura $\mathrm{N}^{\circ} 3$ se ve reflejado el problema de las escalas temporales: no es lo mismo analizar la demanda de tiempo para la escolarización obligatoria de un/a alumno/a a lo largo de un día, una semana, un año o a lo largo de la vida.

Es a principios de la década de 1960 que en geografía se empieza a reflexionar conscientemente sobre el tiempo como algo que afecta a las actividades de los individuos; este paso aparece en George (1974; editado originalmente en 1966) y Randle (1965, 1966), quien reemprende el tema de unos trabajos poco utilizados de Whittlesey (1945) y de Ogilvie (1953). Reflexiones sobre el uso del tiempo en geografía se pueden encontrar en trabajos más recientes (Parkes \& Thrift, 1980; Mendizábal, 1993a; May \& Thrift, 2001; Muñoz Ramírez, 2006).
Figura $\mathrm{N}^{\circ} 3$

Las distintas escalas del tiempo: el tiempo de escolarización obligatoria de un/a alumno/a tipo de España en la actualidad

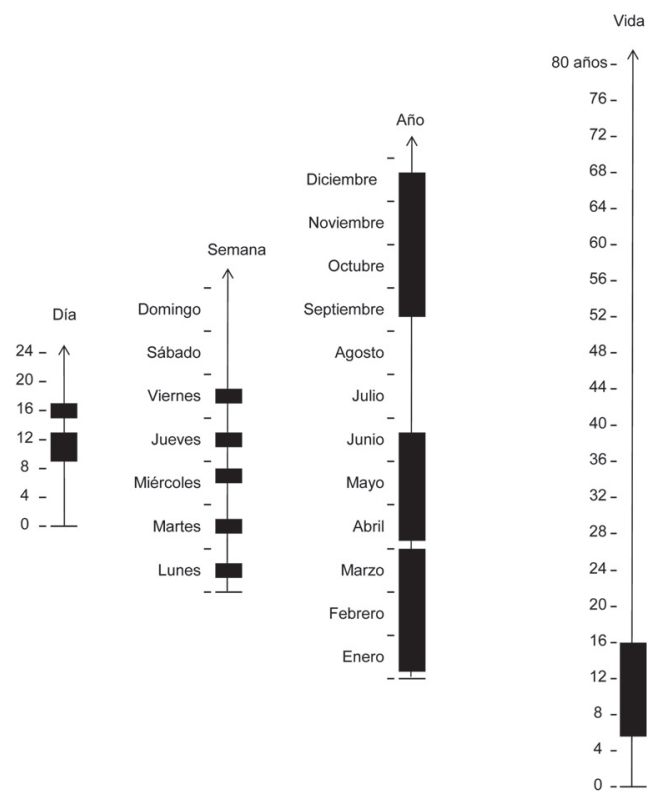

Fuente: adaptación de Enric Mendizábal y Ariadna García a partir de Carlstein (1978: 153). 
Pierre George (1974) escribe que la dimensión del uso cotidiano del espacio la sugiere la frecuencia de uso. El espacio de vida está construido, principalmente, por los lugares de trabajo, de residencia, de ocio, por los lugares donde se realizan las relaciones sociales; el espacio de vida se forma a partir de las repeticiones de ciertos desplazamientos, y cuando el individuo tiene que dar las medidas de su espacio, las da por unidades de tiempo y por la accesibilidad.

Es importante para George (1974: 60-62) diferenciar dos tipos de tiempo en el análisis del uso cotidiano del espacio: el tiempo vulgar y el tiempo anómalo. El estudio geográfico del tiempo vulgar analiza las diferencias de uso del tiempo vivido según el espacio donde se efectúan los actos de los individuos. George propone que la geografía dé una imagen cartográfica y descriptiva de las diferentes rutinas temporales (la diaria, la semanal, la anual) de la población, espacializando los tiempos de vida, según los hábitos que escapan a las leyes naturales. El tiempo anómalo determina una inflexión -un punto de ruptura- en la evolución de la sociedad; esta ruptura puede estar causada por una crisis económica, una guerra, una revolución o una catástrofe natural, entre otras posibilidades.

Pierre George (1974: 65) describe diferentes aspectos del uso tiempo para el análisis geográfico. Así, el tiempo anómalo influye en el espacio y en las relaciones sociales, según la escala de su impacto (universal, continental, regional, local); el tiempo cíclico viene dado por los ritmos naturales, relacionados directamente con las variaciones climáticas a lo largo del año; el tiempo técnico, dominante en las sociedades occidentales, en que el consumo y la producción de bienes y servicios no depende de los ritmos naturales, aunque ciertamente influyen (las vacaciones se acostumbran a hacer durante los meses más calurosos); y la percepción del tiempo, puesto que el individuo encuentra el tiempo más o menos largo según la actividad que hace, aunque la duración cronometrada sea la misma.

Randle $(1965,1966)$ sistematiza como el tiempo se refleja en el espacio, ya sea bajo la forma del cambio, donde se percibe la evolución del paisaje a partir de un tiempo lineal y cronológico, o a partir del movimiento, que Randle identifica con el cambio inmediato y el tiempo cíclico. Se desprende de estos textos de Randle que la interrelación entre el tiempo y el espacio se ve a través de las actividades humanas: Ios ciclos agrícolas, así como el de ciertas industrias extractivas, o ciertas migraciones temporales son consecuencia del régimen climático; los ciclos diarios, que Randle incorpora dentro de la geografía del movimiento, hacen que el uso del espacio sea muy diferente a lo largo de un día (que haya más o menos tránsito en una ciudad, que aumente o disminuya del consumo de agua y de energía...).

Randle (1965, 1966) propone unos conceptos sobre cómo utilizar el tiempo en geografía. Entre ellos cabe destacar el ritmo, entendido como una media de recorrido tipo (la lentitud de las gabarras de un canal, la rapidez de los automóviles en una autopista); la frecuencia, que origina coincidencias y simultaneidades (la hora de entrada al trabajo en el centro de la ciudad); el movimiento entendido como traslado de personas y cosas; la velocidad, ya que en función de ella se puede acceder con más rapidez a los lugares que con otras velocidades se encuentran más lejos.

A principios de los años 70 del siglo $X X$, con los trabajos de Torsten Hägerstrand, el tiempo se integra explícitamente en la geografía humana; Hägerstrand dice que:

"desde aquel momento [1947] intenté... bien, algo me golpeó aquí [en la cabeza], y lo he ido preparando durante mucho tiempo, y era la posibilidad -o la necesidad- de combinar geografía y tiempo -no historia, sino tiempo- en una forma diferente de cómo se había intentado hacer hasta entonces. En lugar de ver el tiempo como una serie de saltos entre momentos, yo intentaba ver los procesos yendo ininterrumpidamente. Esta es en todos sentidos la única nueva idea que he tenido en mi vida, y esto es en lo que he ido trabajando desde entonces." (Hägerstrand y Buttimer, 1985: 14) ${ }^{7}$.

\footnotetext{
7 Este texto de Hägerstrand y Buttimer es la transcripción de una entrevista. Las anotaciones entre corchetes son del autor.
} 
Hägerstrand intenta reflejar procesos sin ruptura en sus trabajos sobre difusión (1952, 1966, 1967), pero una de las críticas recibidas fue que justamente no conseguía dar esta visión de no interrupción. Donde se refleja de una manera clara la idea de ver los procesos sin rupturas es en sus trabajos de time geography, que le permitieron superar la insatisfacción que le producía el enfoque positivista de la geografía teorético-cuantitativa. Hägerstrand trató como punto central la incorporación del tiempo en el análisis geográfico, que hasta el momento se había reducido a intentar mapificar algún índice de los cambios ocurridos entre dos puntos en el tiempo, que es utilizado como una variable discreta. Hägerstrand estudia como los seres humanos utilizan el tiempo y el espacio como recursos escasos para realizar sus quehaceres cotidianos, sabiendo que las herramientas cartográficas de las cuales se disponía eran poco útiles para registrar acontecimientos en coordenadas espacio-temporales, y que, por lo tanto, se tenían que crear estas nuevas herramientas.

Desde la aparición de What about people in Regional Science? (Hägerstrand, 1970), este geógrafo fue construyendo el esquema teórico de la time geography así como su aplicación práctica. La time geography fue una aportación interesante e innovadora dentro de la geografía humana: el centro de este enfoque es el interés por el uso del tiempo y del espacio por parte de los individuos para ayudarlos a conseguir sus objetivos.

Hägerstrand presenta en sus trabajos (1970, 1972, 1974, 1975, 1982) los conceptos básicos de la time geography: trayecto, limitación, dominio, proyecto y diorama, entre otros. El individuo, a lo largo del espacio y del tiempo describe un trayecto que empieza con el nacimiento y acaba con la muerte; los trayectos pueden ser estudiados a lo largo de toda una vida, así como por periodos de tiempos más cortos (Figura $N^{\circ} 3$ ). El trayecto de un individuo o el trayecto de agregados de individuos ayuda a identificar el significado de la continuidad espacio-temporal en la sucesión de situaciones y acontecimientos.

Las limitaciones son hechos que impiden realizar el trayecto tal como querría un individuo o los agregados de individuos. Las hay de tres tipos: de capacidad, de acoplamiento y de autoridad. Las limitaciones de capacidad están derivadas de las necesidades físicas y fisiológicas (la regularidad de dormir y de comer, por ejemplo), así como de los medios de transporte y de comunicación (con un automóvil podemos ir más lejos que a pie en un mismo periodo de tiempo, por ejemplo). Las limitaciones de acoplamiento están definidas por dónde, cuándo y por cuánto tiempo los individuos pueden encontrarse con otros individuos, enseres y materias para reproducirse, producir, consumir o intercambiar: en la tienda, las personas que venden y las que compran forman un haz de trayectos para intercambiar artículos; en una clase, profesorado y alumnado forman un haz de trayectos para intercambiar informaciones e ideas; estos haces están predeterminados por los horarios. Las limitaciones de autoridad tienen que ver con los dominios, espacios que no son accesibles a todo el mundo, ya que se tiene que pagar, luchar o hacer algún tipo de ceremonia para poder acceder (por ejemplo, pagar para entrar en un cine, o enseñar la documentación necesaria para entrar a un Estado por un paso fronterizo).

El proyecto está constituido por el haz del conjunto de actividades necesarias, individuos y objetos que tienen que participar para la realización de cualquier intención o conducta orientada a una finalidad. Cualquier proyecto requiere una serie de trayectorias y de haces de trayectorias para poderse realizar. Hägerstrand añade que para que el proyecto se realice sin muchas interrupciones ni roturas, tendrá que encajarse en un presupuesto de tiempo, es decir, de la cantidad de tiempo que el individuo o agregados de individuos (un ser humano, una familia, una institución...) pueda proveerse y disponer. Este presupuesto de tiempo a gastar en un espacio concreto aparece cubierto por los diversos dominios, los cuales forman una red compleja de entidades que protegen sectores de espacio-tiempo, para así resguardar a los proyectos que se están realizando de interrupciones innecesarias o no deseadas, y para ofrecer reservas de espacio-tiempo para el futuro.

El estudio de una región tendría que hacerse a partir del análisis de una red de haces conectados entre ellos, y ver cómo los 
individuos entran y salen de estos. El diorama sería una nueva manera de describir una área concreta, puesto que reúne el espacio, los proyectos que se dan en él y las relaciones entre los proyectos y el espacio. Hägerstrand (1982) describe el diorama de los años en que él era alumno de escuela primaria, de una zona rural agrícola en la que también había una fábrica de productos metálicos. El uso de los conceptos de trayectoria, proyecto y diorama a través de datos autobiográficos hace que la relación entre la teoría y la práctica sea muy inteligible, pero parece como si este concepto de diorama regresara al concepto de paisaje de la geografía posibilista.

En resumen, la time geography considera el tiempo y el espacio como recursos que apremian la actividad de los individuos, los cuales tienen diferentes posibilidades de movimiento en el espacio (según cual sea su nivel económico, tengan o no tengan coche, el trabajo que desarrollen, las obligaciones sociales...), pero limitados por el tiempo. Las trayectorias, los proyectos, los dominios y los dioramas, sean de individuos o de agregados de estos (familias, empresas, instituciones...) son los temas claves de la time geography, una manera de hacer geografía en la que Hägerstrand busca "un camino para encontrar una coherencia conceptual en el entendimiento del geógrafo del mundo humano, todo el camino que va desde casa hasta el globo y desde el día a toda una vida" (Hägerstrand, 1974: 144-145).

De la descripción bibliográfica de los párrafos anteriores se puede ver un doble trato del tiempo en el campo de la geografía humana: el primero sería el tiempo histórico, que permite analizar los cambios de las características y la evolución de los paisajes y de las localizaciones de los fenómenos y lugares; el segundo sería considerar el tiempo como un recurso escaso, y que disponer -o no- de una buena distribución del tiempo significa que el individuo pueda $-\mathrm{O}$ no- realizar sus actividades y pueda tener -o no- una vida más o menos satisfactoria (Mendizábal, 1993a).

Pero además de mi interpretación personal sobre cómo los geógrafos han utilizado el tiempo en sus estudios, se pueden encontrar otras interpretaciones. Thrift (1977) escribe que el tiempo se puede ver, dentro de la geografía humana, de tres maneras diferentes: como una escalera para que los procesos suban, como un continuo locacional, y como un recurso. En la primera parte de su texto, Thrift trata el tiempo según varias disciplinas: el tiempo en física y filosofía, el tiempo biológico (los ciclos circadianos principalmente), el tiempo en psicología (la percepción del tiempo), la organización temporal de la sociedad... A lo largo de la segunda parte, Thrift trata el tiempo como recurso, haciendo una amplia referencia a los trabajos de la escuela de Lund, relacionada con Torsten Hägerstrand.

Una segunda explicación del uso del tiempo en geografía es la que hacen Bailly y Béguin (1992). Este trabajo hace breves comentarios sobre los diferentes tipos de tiempos (cíclico, meteorológico, teológico, cósmico, social), así como sobre la poca reflexión que se ha hecho de este tema en geografía, a pesar de la aproximación geohistórica de la geografía regional posibilista. En cualquier caso, Bailly y Béguin acaban escribiendo que, para el análisis geográfico, el tiempo se tiene que utilizar como un recurso escaso, igual que el espacio; estas dos variables constituyen un tipo de nicho ecológico que ocupan los individuos. Bailly y Béguin siguen los conceptos de la time geography puesto que permiten comprender las localizaciones y las extensiones de las acciones humanas, sus desplazamientos, el inicio y el fin de las acciones, las frecuencias y los modos de repeticiones temporales y espaciales de las acciones, las secuencias y tipos de actividades.

Un texto relativamente reciente de Muñoz Ramírez (2006) sistematizó la bibliografía reciente sobre cómo se ha utilizado el tiempo en el análisis geográfico. En esta tercera explicación se diferencia el tiempo del territorio de los territorios del tiempo. Por tiempo del territorio, Muñoz Ramírez entiende las diferentes escalas temporales que coinciden en un mismo territorio, que a diferencia de las planteadas en las Figuras $\mathrm{N}^{\circ} 2$ y $\mathrm{N}^{\circ} 3$, clasifica como tiempo histórico (cronológico), laboral (pautado por las actividades productivas) y digital (dirigido por las nuevas tecnologías de la comunicación). Por territorios del tiempo, Muñoz Ramírez entiende el uso del territorio según la intensidad y variabilidad: un mismo territorio no se usa igual de día o de noche, 
un día laborable que un fin de semana. Aquí le da otro nombre al tiempo anómalo de George (1974) y a la geografía del movimiento de Randle $(1965,1966)$ citados anteriormente, y recupera implícitamente los trabajos de la time geography, así como otros realizados por Mendizábal (1993c) y Mendizábal et al. (1994). Otros autores han reflexionado con posterioridad sobre estos territorios del tiempo (Nofre i Mateo, 2009).

En resumen, creo que queda reflejado de manera clara la importancia de tener presente el tiempo como una variable fundamental para hacer un buen análisis geográfico, y, por razones obvias, para hacer una buena geografía histórica que vaya más allá del tiempo cronológico.

\section{¿Qué se estudia (principalmente) en geografía humana?}

Recordemos la definición que he utilizado para definir geografía humana: es el estudio de los movimientos de las personas en la superficie de la Tierra a lo largo del tiempo. En las páginas anteriores se han podido ver distintas maneras de tratar el tiempo en geografía humana. En las páginas siguientes veremos algunos ejemplos de trabajos en los que se pueden observar algunas maneras del uso del tiempo en geografía (que se pueden identificar sin excesivos problemas como geografía histórica). Para ordenar estos trabajos he elegido una tipología de conceptos temporales: cambio, evolución, transformación y proceso, los cuales, obviamente, están interrelacionados y son casi sinónimos, como lo pueden ser los conceptos geográficos de paisaje, región, espacio, territorio o lugar. Elegir un orden en la presentación de los cuatro conceptos temporales que propongo es completamente arbitrario, ya que no hay una única jerarquía lógica para describirlos y explicarlos. Pero el orden elegido es el siguiente $^{8}$.

8 Las definiciones de los conceptos cambio, evolución, transformación y proceso se basan en los diccionarios y textos habituales, entre los cuales el diccionario de la Real Academia de la Lengua Española (http://www.rae.es) y el diccionario de

\section{Cambio}

Si por cambio entendemos la alteración de la realidad a través de la modificación de sus atributos, convirtiendo la realidad en otra realidad, los trabajos de geografía en los que se presentan una sucesión cronológica de distintos estadios (cross sections) son un buen ejemplo, ya que utilizan el tiempo como una variable discreta.

El cambio se hace evidente en los modelos de difusión. La difusión se puede definir como la propagación de un fenómeno en el espacio y en el tiempo, y como objeto de estudio ha sido un tema habitual en geografía: dentro de la geografía histórica ya se había estudiado como las migraciones (entendidos como el agente difusor) ocupaban nuevos territorios, y dentro de la geografía cultural como se trataba de la difusión de aspectos culturales (religión, lenguas, tradiciones...) así como de aspectos técnicos (enseres, objetos, técnicas...). La difusión de información, conocimientos, enseres y bienes solamente es posible si existe una red por donde puedan moverse los agentes difusores (McNeill y McNeill, 2004). Así, la difusión es un cambio en el tiempo de dos dimensiones: la social y la espacial.

Hägerstrand (1952, 1966, 1967) se interesó por el tema de la difusión de las innovaciones (ya sea de actitudes, ya sea de técnicas) en la Suecia rural en la primera mitad del siglo XX. Disponiendo de datos precisos de unas determinadas variables (la adquisición de automóviles, la introducción de nuevos cultivos, la adopción de medidas contra la tuberculosis vacuna...) elaboró mapas que muestran la progresión del cambio en el territorio estudiado. Esta elaboración se hace por simulación: con la ayuda de un ordenador, Hägerstrand reprodujo la secuencia de los acontecimientos y reconstruyó las etapas de la evolución (a partir de unas hipótesis para

la lengua catalana (mi lengua materna) del Institut d'Estudis Catalans (http://dlc.iec.cat), así como los diccionarios y textos siguientes: Agnew y Duncan (2011); Bailly (1998); Brunet et al. (1993); De Vecchis y Palagiano (2003); dell'Agnese (2009); Ferrater Mora (2009); Gregory et al. (2009); Holloway et al. (2004). 
el buen funcionamiento del modelo) para explicar cómo se llega a la situación actual. Una aplicación de este tipo de estudio se puede encontrar en Tulla (1983), quien desde una perspectiva marxista utiliza los modelos de difusión propuestos por Hägerstrand para explicar la transformación geoeconómica de la zona montañosa del Pirineo de Cataluña (región del nordeste de España) a lo largo del siglo XX.

Los modelos de difusión de las innovaciones presentan los cambios en el espacio en intervalos constantes de tiempo, lo que evidentemente es poco real, pero no hay otras posibilidades metodológicas para representar estos cambios. En cualquier caso, los modelos de difusión elaborados por Hägerstrand son un ejemplo muy interesante de la interacción entre un proceso temporal y una forma espacial.

Otros ejemplos de cambio en geografía se encuentran en el libro editado por Chorley y Haggett (1971), una compilación de trabajos en los que los distintos autores hacen una selección y unas reflexiones sobre los modelos que se han utilizado dentro de distintas subdisciplinas geográficas. En el capítulo introductorio, Chorley y Haggett explican la evolución de la historia de la geografía a partir del concepto de paradigma (Kuhn, 1962) y presentando una clasificación de los diferentes tipos de modelos. Desde la perspectiva de cambio, entre los modelos de desarrollo económico destaca el de Rostow sobre las etapas del crecimiento económico. Asimismo, el modelo de Taaffe, Morrill y Gould explica el proceso de creación de una estructura moderna de localización industrial en el que hay cuatro etapas sucesivas.

En referencia a los modelos de poblamiento, el cambio se identifica con el paso del tiempo (que Haggett [1976] denomina evolución histórica), el cual hace variar la jerarquía de los lugares centrales, algo que no tienen en consideración los modelos de poblamiento de Christaller y de Lösch ( $\mathrm{Ha}$ ggett, 1976: 125), pero que sí hacen algunos geógrafos posteriores como Carter (1966). El modelo del rango-tamaño permite comparar la jerarquía en la distribución del poblamiento en diferentes momentos históricos (Vries, 1987).
La principal limitación de la geografía del cambio en relación con el tiempo es que solamente explica los cambios/transformaciones a partir de la técnica de cross sections que se suceden en etapas temporales sucesivas y homogéneas. El tiempo aparece como una variable discreta, cuando el uso que se hace cotidianamente del tiempo es de variable continua.

\section{Evolución}

Si se ha identificado el cambio geográfico con el uso de un tiempo matemáticamente discreto, la investigación geográfica que trata el tiempo de manera continua es la que está incluida en este apartado. En geografía se acostumbra a identificar evolución con el cambio gradual de calidad y/o cantidad de una/s variable/s a lo largo del tiempo. Por ejemplo, se investiga la evolución de la población, como también la evolución de un paisaje. La geografía regional posibilista sería un claro ejemplo de este tipo de trabajos geográficos. Uno de los textos teóricos fundamentales de esta manera de hacer geografía es The Morpholgy of the Landscape, de Sauer (1925), quien explica que el paisaje actual es el resultado de la interacción entre un grupo humano y el medio natural donde habita, teniendo presente que es la cultura de este grupo la que determina la evolución del paisaje.

Los libros sobre geografía histórica de Europa de East (1943), Samarkin (1976), Dodgshon (1987) y Pounds (2000) hacen una interpretación de la evolución de las características geográficas de Europa a lo largo del tiempo, en períodos largos y con perspectivas ideológicas distintas. East representa la geografía clásica la primera mitad de siglo XX, explicando la geografía del poblamiento, la geografía política y la geografía económica entre el imperio romano y finales del siglo XIX. Samarkin, utilizando el marxismo ortodoxo de la guerra fría, utilizando un esquema temático y cronológico muy similar al de East, explica la evolución de la geografía histórica europea desde una presunta perspectiva crítica. Esta perspectiva crítica sí que se encuentra en el libro de Dodgshon, quien ofrece una interpretación de la evolución de la geografía histórica europea relacionando los diferentes estadios del desarrollo de la sociedad con los distintos sistemas de orden 
espacial existentes, explicando la evolución de Europa desde las sociedades cazadorasrecolectoras hasta el capitalismo globalizado. Pounds vuelve a presentar una evolución de la geografía histórica europea descriptiva como la de East, eso sí, con nuevas informaciones y datos que se han cartografiado en numerosos mapas.

En el marco europeo, Planhol y Claval (1988), Rombai (2002) y Whyte (2002) presentan una evolución del espacio francés, italiano e inglés respectivamente desde antes de la romanización hasta el presente. En uno de los mejores textos de geografía histórica para mi gusto que he leído nunca, Pedro Cunill (1999) hace una más que interesante explicación geohistórica de la evolución del conjunto de América Latina.

A una escala paisajística más grande, se puede citar el libro editado por Esteban (2003), donde los geógrafos e historiadores que participaron en el libro han utilizado toda una serie de fuentes (análisis de textos y fotografías, observación directa, entrevistas en profundidad semiestructuradas, palinología y antracología...) para realizar una geohistoria ambiental del paisaje del Pirineo de los últimos siete mil años. Un trabajo donde hay geografía física, humana y regional integrada para analizar y describir la evolución de una parte de la superficie terrestre.

\section{Transformación}

Por tal concepto, aquí se entienden las rupturas en la historia que suponen una transformación de forma que alguien o algo se transmute en algo, otra cosa distinta, que provoque una transformación en la estructura de lo que se estudia y analiza.

Si hay un texto básico en historia (geografía histórica) social sobre la transformación de la relación entre tiempo y sociedad, este no es otro que Tiempo, disciplina de trabajo y capitalismo industrial de E.P. Thompson (1995). En este texto se parte de la idea que entre 1300 y 1650 hay unas transformaciones importantes en la percepción del tiempo en Europa occidental, que permiten el paso de un trabajo con ritmos irregulares (característicos de una economía preindustrial) a un trabajo donde el ritmo tiene que ser sincro- nizado hasta el más mínimo detalle (típico del fordismo). Por esta razón, en el período inicial de la industrialización se produce una difusión general de los relojes, ya que la Revolución Industrial exige una gran sincronización del trabajo (Thompson, 1995: 413). Se debe de tener presente que, con el trabajo asalariado, el tiempo se convierte en moneda: el tiempo no pasa sino que se gasta. Asimismo, Thompson muestra las resistencias de la gente a trabajar y vivir bajo esta presión del tiempo medido hasta el último instante. Thompson reflexiona sobre qué hacen los trabajadores cuando no trabajan: pasan el rato, y esto es peligroso, porque pueden pensar y relacionarse con otras personas, y no solamente dedicarse a consumir en las unidades de tiempo de no trabajo.

En el capitalismo se quiere ahorrar tiempo, está mal visto "perder el tiempo". Hay ejemplos muy interesantes de cómo esta necesidad de no "perder el tiempo" hace cambiar la cotidianidad. En la Bretaña francesa, la nueva política de productividad agrícola hizo reagrupar las parcelas dispersas de los campesinos para disminuir las idas y venidas, así como permitir el uso del material agrícola moderno sobre grandes superficies. Esta concentración parcelaria destruyó las vallas de brezos, el microclima se convirtió en más riguroso al ampliar los efectos del frío, la sequía y el calor, y modificó el reparto de las aguas subterráneas (Chesneaux, 1984: 143144).

Allan Pred (1986), utilizando la metodología de la time geography, estudia y analiza la transformación del sur de Suecia, la región de Escania, a partir del paso de un modo de producción doméstico y artesano a otro donde la fábrica y los grandes almacenes dominan la vida cotidiana. Pred centra su atención en la alteración de los proyectos de producción, y por lo tanto, en los trayectos que tienen que realizar para conseguir los proyectos, así como en el impacto de la vida cotidiana familiar y la participación en el tiempo libre. Pred también muestra cómo el paso de una organización de la propiedad de campos abiertos (open fields) a campos cerrados entre 1750 y 1850 en Escania, a partir de un proyecto institucional, interfiere en las trayectorias individuales ( $y$ en sus proyectos) transformando la percepción que tenían estos 
individuos del lugar, así como también se transforman las estructuras de las relaciones sociales. Este lenguaje teórico se transforma en algo real cuando se ve que la propiedad de un campesino que antes de la intervención del Estado era de diversas pequeñas parcelas alejadas entre ellas se convierte en una única gran parcela. En la primera situación, vivir en una aldea era la mejor situación para todos los campesinos, ya que era el lugar central más o menos cercano a todas las parcelas. Cuando solamente se tiene una parcela grande, la mejor localización es tener la vivienda en el centro de la parcela: se pasa de un poblamiento agrupado a uno de disperso, desaparece toda la red de pequeños caminos que debían permitir acceder a todas las parcelas, desaparece la vida social de la aldea donde todo el mundo podía compartir las tardes y las noches después del trabajo. Una enorme transformación por tal de no perder tiempo para ir andando a las distintas parcelas y aprovechar más el tiempo para trabajar y producir. Pred demuestra cómo el lugar es un proceso históricamente contingente que asegura la interdependencia entre lo social y lo espacial: los seres humanos producen la historia y los lugares y, al mismo tiempo, son producidos por estos. El lugar limita $y$, al mismo tiempo, permite nuestras acciones; a su vez nuestras acciones construyen y mantienen los lugares (Albet, 2001: 40).

\section{Proceso}

Por proceso entiendo el conjunto de acciones, operaciones e interacciones entre individuos y grupos sociales que, a través de un conjunto de fases sucesivas, suponen un cambio caracterizado por una serie de características comunes que están dirigidas a una finalidad definida. Los trabajos de geografía histórica que se incluyen en este apartado son los que trabajan la geohistoria de la globalización del capitalismo ${ }^{9}$. Un problema para realizar este tipo de estudios es que en cualquier proceso suceden a la vez muchas cosas, pero dicho proceso solamente se puede explicar, cuando hablamos o escribimos,

\footnotetext{
9 El término globalización procede del inglés, y es el que se ha adoptado en la mayoría de idiomas (incluido el castellano) y escuelas de pensamiento. En francés se ha adoptado el término mundialización.
}

siguiendo un orden sucesivo que está muy lejos de la simultaneidad en la que suceden las cosas. La pregunta es la siguiente: ¿en qué orden explicamos lo que coincide en el tiempo? Parece obvio que la explicación del proceso será diferente según el orden narrativo escogido, ya que se hará un énfasis distinto en cada uno de los temas que participan en el proceso.

El éxito del capitalismo, el sistema territorial, económico, cultural, político y social actualmente dominante en el conjunto del mundo, es el resultado del proceso de globalización acaecido en el mundo a lo largo de los últimos 250 años e iniciado con la llegada a América de castellanos y portugueses, los cuales también van a Asia del sudeste. El crecimiento del conjunto de la población mundial (la transición demográfica como modelo explicativo), el cambio en el poblamiento (el paso de vivir en ámbitos rurales y en núcleos de población pequeños que aprovechaban al máximo la explotación del territorio cercano, a vivir en espacios urbanos de todo tipo interrelacionados para trasladar todo tipo de cosas), el cambio en la movilidad (en la actualidad podemos ir a cualquier lugar para efectuar cualquier actividad, y si no vamos, podemos comunicarnos en el acto), la(s) revolución(es) agrícola(s) del mundo moderno (desaparición del barbecho, nuevos cultivos, nuevas técnicas) y la(s) revolución(es) industrial(es) son los elementos de un proceso que hace que se pase de un mundo donde la mayoría de la población vive y trabaja alrededor de un mundo rural y agrario a un mundo urbano e industrial, y actualmente a un mundo metropolitano y de servicios.

Así, a partir del siglo XVIII suceden una serie de procesos a la vez (creo que es imposible saber qué fue causa de qué), que hacen que cada vez más cualquier parte del mundo se interrelacione más con el resto del mundo, y que los que no lo hacen (por las razones que sean) quedan al margen. Blaut (1993, 2000), Ferro (2000), Frank (2008), Goody (2004), Guha (2003), Iniesta (1998, 2000), Izard (2000), Linebaugh y Rediker (2005), Pipitone (2003), Wallerstein (1979, 1984, 1999) y Wolf (1987) explican el éxito de la difusión del capitalismo desde perspectivas explícitamente no eurocéntricas, y destacando la importante resistencia (militar, ideológica, 
cultural) de los pueblos no europeos frente a la invasión e imposición del capitalismo europeo. Otros autores explican este proceso mostrando los procesos que han sucedido en las diferentes áreas culturales, como Braudel (1966), Fossaert (1994), Grataloup (2007) o Jones (1990, 1997), pero sin hacer una referencia ideológica explícita de los conflictos existentes. Y finalmente, hay autores que destacan el éxito del capitalismo gracias a las características especiales y privilegiadas de los europeos, como Landes (1999) o Huntington (1997), este último de forma excesiva por sus falacias ideológicas pro occidentales. Un reciente atlas (Denis y Greilsamer, 2011) muestra un conjunto de mapas y textos muy sugerentes sobre este largo proceso de globalización, en el que se recoge una visión crítica y propositiva del proceso. A continuación se mostrarán tres tipos de explicaciones geohistóricas del proceso de globalización y una reflexión final sobre este proceso centrado en Brasil.

Alfred W. Crosby (1986) explica los aspectos biológicos que han ayudado al capitalismo a tener éxito en su proceso de difusión, centrándose en las plantas, los animales y las enfermedades. La difusión de las plantas europeas por el continente americano fue un proceso básico para la supervivencia de la población de origen europeo, pero también para la destrucción y desaparición del paisaje previo. Y no solamente por la llegada de las malas hierbas europeas, sino también por las plantas forrajeras, los frutales y los cereales, que aparecen con los europeos. Estas plantas les permiten su supervivencia, pero además implican el cambio del patrón de cultivos de los americanos, ya que sus productos tradicionales -a excepción del maíz y la patata- se producen cada vez más en territorios más marginales y marginados. Los animales domésticos europeos como los "caballos, ganado vacuno, cerdos, cabras, ovejas, asnos, gallinas, gatos y demás [...tienen una...] eficacia y rapidez [...] de alterar el medio, incluso a nivel continental, [...] superiores a las de cualquier máquina que haya podido inventarse, debido a que estos animales pueden autorreproducirse" (Crosby, 1986: 193-194). Los ejemplos de cómo los bovinos en América y los ovinos en Australia acaban con los pastos autóctonos que son sustituidos por las malas hierbas europeas son clásicos para la biogeografía cultural. Además de los malos tratos, sobreexplotación y hambrunas provocados por los europeos, la población indígena de las colonias europeas padeció una serie de enfermedades contra las cuales no tenían defensas, y, además, las difundieron conscientemente regalando mantas utilizadas por europeos infestados de estas enfermedades. Crosby define el proceso de expansión biológica de Europa como un intercambio desigual.

Peter J. Taylor (1999) explica el mundo desde el siglo XVII hasta la actualidad a partir de tres regiones que son las iniciales de tres etapas geohistóricas de la modernidad: Ios Países Bajos de la época mercantil, la Gran Bretaña de la revolución industrial, los Estados Unidos de América de la revolución del consumo. Aunque en su libro hay multitud de referencias constantes de diversos temas geográficos (economía, poblamiento, sociedad, movilidad...), Taylor se centra sobre todo en los aspectos políticos que generan la modernidad. Por ejemplo, en el capítulo 3 explica, entre otras cosas, cómo la pintura en los Países Bajos, la novela en Gran Bretaña y el cine popular en Estados Unidos muestran cómo debe ser una vida cotidiana políticamente correcta en cada uno de los momentos históricos a los que Taylor se refiere anteriormente. En el capítulo 4 se hace hincapié en los distintos tipos de Estado; en el 5 los movimientos políticos, mostrando algunos de los movimientos alternativos y críticos con el sistema dominante (socialismo en Gran Bretaña, ambientalismo en Estados Unidos); en el capítulo 6 se tratan las tensiones geográficas en los Estados y en las familias; finalmente, en el capítulo 7 se trata de la americanización del mundo. Así, Peter J. Taylor explica el proceso de la geohistoria de la globalización desde una perspectiva geopolítica de los últimos 400 años.

Peter Hall y Paschal Preston (1990) parten de los ciclos de Kondratieff, los cuales sugieren que el capitalismo tiene un "mecanismo automático endógeno que daba por supuesto que la economía capitalista había de regenerarse de todas sus crisis sucesivas" (Hall y Preston, 1990: 34). Los ciclos de Kondratieff se definen como unas oscilaciones de desarrollo económico que tienen una periodicidad de 40-60 años, con una primera etapa de 
crecimiento y una segunda de un cierto declive. Mientras que los datos empíricos macroeconómicos justifican con poca dificultad la existencia de estos ciclos, no ocurre lo mismo con la teoría económica, ya que no hay fórmulas matemáticas que puedan justificar estos ciclos. Así las cosas, la existencia real de los ciclos de Kondratieff permite a Hall y Preston utilizar los ciclos de Kondratieff para relacionarlos con los procesos de difusión de las innovaciones.

Moraes (2009) presenta una compilación de materiales diversos de geografía histórica del Brasil con una lógica interna muy bien construida. A partir de los conceptos de soberanía, territorio e identidad nacional, Moraes explica cómo, desde la geografía crítica postcolonial, se puede estudiar el proceso de formación de una parte de la superficie terrestre que acabará siendo Brasil, un territorio que, según Moraes, forma parte de la periferia del sistema capitalista global actual. La colonización de lo que después será Brasil por parte de los portugueses entre los siglos XVI y XVIII tiene una explicación exclusivamente geopolítica: evitar que otros (españoles, franceses, holandeses...) se queden con este territorio. La discusión alrededor de los conceptos territorio, región, espacio económico y proceso de colonización es muy rica, y permite explicar la estructura territorial del Brasil en el período de la independencia: en 1822, cuando se proclama el imperio, Brasil es un territorio utilizado en forma de archipiélago: regiones de plantaciones, redes urbanas y ciudades creadas por la minería, áreas de ganadería extensiva, núcleos de colonización relacionados con alguna mercancía puntual, enclaves en las áreas de frontera (misiones, campamentos militares) y amplias zonas de dominio formal no incorporadas por el proceso colonizador. Con todos estos elementos, la acción política estatal tendrá que construir la identidad, la nación y la nacionalidad brasileña. Y para hacer una geografía histórica del Brasil, Moraes escribe que es necesario caracterizar los ámbitos naturales existentes, los espacios construidos, los espacios de generación de valor económico a partir de la producción de bienes, la logística de las infraestructuras y de las necesidades energéticas. Para hacer todo esto, se necesita conocer los procesos geohistóricos que han llevado a la situación actual. Por esta razón, Moraes hace una inter- pretación geográfica del Brasil a partir de los postulados de la nueva geografía regional.

Para acabar este apartado solamente queda tener presente la dificultad que tiene la Geografía para explicar procesos temporales. Y aquí se han querido señalar algunas de las distintas posibles maneras de explicar un mismo proceso geográfico, a saber, la difusión y éxito de la globalización, es decir, del capitalismo, donde el tiempo y la historia, entendidos como el cambio, la evolución, la transformación y los procesos son elementos clave.

\section{¿Por qué se estudia la geografía humana de esta manera?}

Paul Claval (1987) escribió que hasta la década de 1970, la geografía mostraba tres tipos de homo. El homo historicus era el apropiado para aquellos geógrafos y geógrafas que creen que la naturaleza humana varía con el tiempo, ya que se trata de un ser humano que es el producto del medio en el que ha nacido y vivido, es consciente de sus limitaciones y las padece, y quiere escapar de lo que le oprime. El homo roboticus es un puro producto de su entorno, y puede describirse como una máquina programada con un programa que se interioriza mediante el proceso de aculturación y socialización, y sabe que si desea conseguir la posición con la que sueña tiene que actuar de manera que satisfaga las expectativas de sus interlocutores. Finalmente, el homo rationalis tiene dos facultades: entendimiento y voluntad; en el momento de nacer tiene la razón ya formada, y ya puede tomar las elecciones más correctas, no le debe nada al entorno ni a la educación. Parece como si la información fuera gratuita para todas las personas y que todas estas tuvieran la capacidad para tratarla y explotarla convenientemente. Todas las personas, situadas en las mismas condiciones y teniendo que solucionar la misma cuestión, la resuelven de una forma idéntica, y además la resuelven de una manera perfecta.

Es a partir de la geografía humanista que aparece un nuevo modelo de homo en geografía, un homo que tiene un cuerpo (con unas capacidades físicas y unas percepciones), que tiene un espíritu modelado por la existencia, con una libido que lo impulsa a ser activo y 
satisfacer todas sus necesidades; la personalidad no está formada solo por el instinto, ya que también hay una elaboración consciente y voluntaria. En la geografía se descubre por primera vez el sentido de los destinos individuales: se exploran las biografías de las personas a las que se quiere comprender, e intenta percibir en cada etapa los horizontes que tienen en frente. La dimensión de la duración, algo que faltaba de manera evidente en la geografía anterior a los años 1970 según Claval, es una de las dimensiones que se deben trabajar para conseguir introducirla en la geografía (Claval, 1987: 242-268). Lo que demandaba Claval hace 25 años, introducir al nuevo homo en los trabajos de geografía, la nueva geografía histórica y cultural crítica ya lo ha conseguido (Boulanger Trochet, 2005; Sunyer Martín, 2010; Zusman, 2006).

Se desprende tanto de algunos de los trabajos citados en el presente artículo como de algunos de los que aparecen en las bibliografías de los tres textos recién citados, que uno de los temas básicos que debe trata la geografía humana es cómo se debería de organizar la sociedad y cómo debería de estructurarse el modelo de poblamiento para asegurar una existencia que pueda ser vivible diariamente y así conseguir una mejor calidad de vida para todas las personas. Las reflexiones sobre las interrelaciones entre tiempo y espacio que se han descrito y analizado en las páginas anteriores son una buena muestra de cómo desde la geografía histórica se puede avanzar por este camino.

Albet (2001: 46) escribe que se quiere evidenciar cómo los procesos que se explican desde la geografía humana, ya sea desde perspectivas teóricas o desde estudios empíricos, sirven para explicar los cambios, evoluciones, transformaciones y procesos geohistóricos que suceden en cada paisaje, región, espacio, territorio y/o lugar, "en una combinación múltiple y compleja de procesos singulares e irrepetibles". El interés de la geografía debería interesarse tanto por el proceso de configuración histórica y geográfica como por la parte de la superficie terrestre donde sucede dicho proceso. Y, especialmente, por las personas que viven en cada parte de la superficie terrestre que se analiza.

Quienes nos dedicamos a la geografía histórica sabemos que tenemos una ventaja respecto las otras personas que hacen geografía: conocemos perfectamente el final del cambio, evolución transformación y proceso que estudiamos. Y si lo hacemos desde una perspectiva crítica, nuestra obligación es decir que el presente no nos gusta de manera suficiente, y que queremos transformarlo. Moraes (2009, capítulo 7) reclama que nosotros, las personas que vivimos en la periferia, no estamos fuera de la historia, y justamente porque nos encontramos en la periferia podemos hacer una investigación y reflexión geográfica interesante, útil y crítica. Este texto de Moraes debería de ser de lectura obligatoria para todas las personas que forman parte del mundo universitario, entre las cuales las que nos dedicamos a la geografía histórica, ya que es una guía para pensar en la transformación del mundo actual.

\section{Referencias bibliográficas}

AGNEW, J.A. \& DUNCAN, J.S. (editors). The Wiley-Blackwell Companion to Human Geography. Londres: Wiley-Blackwell, 2011.

AGNEW, J.; LIVINGSTONE, D.N. \& ROGERS, A. (editors). Human Geography: an essential anthology. Londres: Blackwell, 1996.

ALBET, A. ¿Regiones singulares y regiones sin lugares? Reconsiderando el estudio de lo regional y lo local en el contexto de la geografía postmoderna. Boletín de la Asociación de Geógrafos Españoles, 2001, No 32, p. 3552.

BAILLY, A. Les concepts de la géographie humaine. París: Armand Colin, 1998.

BAILLY, A. y BÉGUIN, H. Introducción a la geografía humana. Barcelona: Masson, 1992.

BLAUT, J.M. The Colonizer's Model of the World. Geographical Diffusionism and Eurocentric History. Nueva York: Guilford, 1993.

BLAUT, J.M. Eight Eurocentric Historians. Nueva York: Guilford, 2000.

BOULANGER, P.H. \& TROCHET, J.R. (directores) Où en est la géographie historique? París: L'Harmattan, 2005. 
BRAUDEL, F. Histoire et sciences sociales: la longue durée. Annales E.S.C., 1958, No 4, p. 725-753.

BRAUDEL, F. Las civilizaciones actuales. Estudio de historia económica y social. Madrid: Tecnos, 1966.

BRUNET, R.; FERRAS, R. \& THÉRY, H. Les mots de la géographie. Dictionaire critique. Montpellier-París: Reclus-La Documentation Française, 1993.

CARLSTEIN, T. Innovation, time allocation and time-space packing. In: CARLSTEIN, T.; PARKES, D. \& THRIFT, N. (editors). Timing space and spacing times. Vol 2: Human activity and time geography. Londres: Edward Arnold, 1978, p. 146-161.

CARTER, H. The towns of Wales. A study of urban geography. Cardiff: University of Wales Press, 1965.

CASASSAS, LI. L'organització territorial de Catalunya. El cas del Solsonès-Cardener. Cardener, 1988, № 5, p. 35-54.

CHESNEAUX, J. ¿Hacemos tabla rasa del pasado? A propósito de la historia y de los historiadores. Madrid: Siglo XXI, 1984.

CHORLEY, R. y HAGGETT, P. (editores). La geografía y los modelos socio-económicos. Madrid: Instituto de Estudios de Administración Local, 1971.

CLAVAL, P. Geografía humana y económica contemporánea. Madrid: Akal, 1987.

CROSBY, A.W. Imperialismo ecológico. La expansión biológica de Europa, 900-1900. Barcelona: Crítica, 1986.

CUNILL, P. La geohistoria. En: CARMAGNANI, M.; HERNÁNDEZ CHÁVEZ, A. y ROMANO, R. (editores). Para una historia de las Américas I. Las estructuras. México DF: Fondo de Cultura Económica, 1999, p. 13-159.

DE VECCHIS, G. \& PALAGIANO, C. (coordinadores) Le parole chiave della geografia. Roma: Carocci, 2003.
DELL'AGNESE, E. (editora). Geo-Grafia. Strumenti e parole. Milán: Unicopli, 2009.

DENIS, J.P. y GREILSAMER, L. El atlas de las mundializaciones de Le Monde Diplomatique. Valencia: Mondiplo, 2011.

DODGSHON, R.A. The European Past. Social Evolution and Spatial Order. Londres: Macmillan, 1987.

EAST, W.G. An historical geography of Europe. Londres: Methuen, 1943.

ESTEBAN, A. (coordinador). La humanización de las altas cuencas de la Garona y las Nogueras (4500 aC - 1955 dC). Madrid: Ministerio de Medio Ambiente, 2003.

FERRATER MORA, J. Diccionario de filosofía. Barcelona: Ariel, 2009.

FERRO, M. La colonización. Una historia global. México DF: Siglo XXI, 2000.

FOSSAERT, R. El mundo en el siglo XXI. Una teoría de los sistemas mundiales. México DF: Siglo XXI, 1994.

FRANK, A.G. Re-Orientar. La economía global en la era del predominio asiático. Valencia: Publicacions de la Universitat de València, 2008.

GARCÍA RAMÓN, M.D. (editora). Teoría y método en la geografía humana anglosajona. Barcelona: Ariel, 1985.

GEORGE, P. Sociología y geografía. Barcelona: Península, 1974.

GOODY, J. Capitalismo y modernidad: el gran debate. Barcelona: Crítica, 2004.

GOULD, P. The geographer at work. Londres: Routledge \& Kegan Paul, 1985.

GRATALOUP, Ch. Géohistorie de la mondialisation. Le temps longue du Monde. París: Armand Colin, 2007.

GREGORY, D.; JOHNSTON, R.; PRATT, G.; WATTS, M.J. \& WHATMORE, S. (editores). 
The Dictionary of Human Geography. Chichester: Wiley-Blackwell, 2009.

GUHA, R. La historia en el término de la historia universal. Barcelona: Crítica, 2003.

HÄGERSTRAND, T. The propagation of innovation waves. Lund Studies in Geography, 1952, Ser. B., No 4. In: WAGNER, P.L. \& MIKESELL, M.W. (editors) Readings in Cultural Geography. Chicago: Chicago University Press, 1962, p. 355-368.

HÄGERSTRAND, T. Aspects of the spatial structure of social communication and the diffusion of information. Regional Science Association, Papers and Proceedings, 1966, No 16 , p. $27-42$.

HÄGERSTRAND, T. Innovation diffusion as a spatial process. Chicago: Chicago University Press, 1967.

HÄGERSTRAND, T. What about people in regional science? Papers of Regional Science Association, 1970, No 24, p. 7-21.

HÄGERSTRAND, T. The impact of social organization and environment upon the timeuse of individuals and households. Plan (especial issue), 1972, p. 24-30.

HÄGERSTRAND, T. Survival and Arena. On the life-history of individuals in relation to their geographical environment. The Monadnock, 1974, No 49, p. 9-29. In: CARLSTEIN, T.; PARKES, D. \& THRIFT, N. (editors). Timing space and spacing times. Vol 2: Human activity and time geography. Londres: Edward Arnold, 1978, p. 122-145.

HÄGERSTRAND, T. El terreno propio de la geografía humana. En: CHORLEY, R. (editor). Nuevas tendencias en geografía. Madrid: Instituto de Estudios de Administración Local, 1975, p. 103-135.

HÄGERSTRAND, T. Diorama, Path and Project. Tijdschrift voor Economische en Sociale Geographie, 1982, No 73, p. 332-339.

HÄGERSTRAND, T. \& BUTTIMER, A. Dialogue Project, Transcript Series G9. Lund: Lund Universitet Reprocentralen, 1985.
HAGGETT, P. Análisis locacional en la geografía humana. Barcelona: Gustavo Gili, 1976.

HAGGETT, P. La geografía, una síntesis moderna. Barcelona: Omega, 1988.

HAGGETT, P. Peter Robin Gould, 1932-2000. Annals of the Association of American Geographers, 2003, Vol. 93, No 4, p. 925-934.

HALL, P. y PRESTON, P. La ola portadora. Nuevas tecnologías de la información y geografía de las innovaciones 1846-2003. Madrid: Fundesco, 1990.

HARVEY, D. Explanation in Geography. Londres: Edward Arnold, 1969.

HOLLOWAY, S.L.; RICE, S.P. \& VALENTINE, G. (editors). Key Concepts in Geography. Londres: Sage, 2004.

HUNGTINGTON, S.P. El choque de civilizaciones y la reconfiguración del orden mundial. Barcelona: Paidós, 1997.

INIESTA, F. Kuma. Historia del África negra. Barcelona: Bellaterra, 1998.

INIESTA, F. Emitai. Estudios de historia africana. Barcelona: Bellaterra, 2000.

IZARD, M. El rechazo a la civilización. Sobre quienes no se tragaron que las Indias fueron esa maravilla. Barcelona: Península, 2000.

JOHNSTON, R.J. Geography and geographers. Anglo-american human geography since 1945. Londres: Edward Arnold, 1985.

JONES, E.L. El milagro europeo. Entorno, economía y geopolítica en la historia de Europa y Asia. Madrid: Alianza, 1990.

JONES, E.L. Crecimiento recurrente. EI cambio económico en la historia mundial. Madrid: Alianza, 1997.

KUHN, T.S. The Structure of Scientific Revolutions. Chicago: University of Chicago Press, 1962.

LACOSTE, Y. La geografía, un arma para la guerra. Barcelona: Anagrama, 1977. 
LANDES, D.S. La riqueza y la pobreza de las naciones. Por qué algunas son tan ricas y otras son tan pobres. Barcelona: Crítica, 1999.

LINEBAUGH, P. y REDIKER, M. La hidra de la revolución. Marineros, esclavos y campesinos en la historia oculta del Atlántico. Barcelona: Crítica, 2005.

MAY, J. \& THRIFT, N. (editors) TimeSpace: geographies of temporality. Londres: Routledge, 2001.

McNEILL, J.R. \& McNEILL, W.H. Las redes humanas. Una historia global del mundo. Barcelona: Crítica, 2004.

MENDIZÁBAL, E. El temps en geografia humana. In: Professor Lluís Casassas. Geografia $i$ territori. Barcelona: Publicacions de la Universitat de Barcelona, 1993a, p. 53-61.

MENDIZÁBAL, E. Lluís Casassas i Simó (1922-1992): geògraf i humanista. Documents d'Anàlisi Geogràfica, 1993b, № 23, p. 97-110.

MENDIZÁBAL, E. L'ús temporal del territori: I'espai de vida dels habitants de la Regió Metropolitana de Barcelona. Barcelona: Enquesta Metropolitana de Barcelona 1990, Institut d'Estudis Metropolitans de BarcelonaMancomunitat de Municipis de l'Àrea Metropolitana de Barcelona-Diputació de Barcelona, 1993c.

MENDIZÁBAL, E.; AJENJO, M.; BLANES, A. \& SÁNCHEZ, E. La població estacional en els municipis de Catalunya. Papers de Demografia, 1994, № 75.

MORAES, A.C.R. Geografia histórica do Brasil: cinco ensaios, uma proposta e uma crítica. São Paulo (Brasil): Annablume Editora, 2009.

MUÑOZ RAMíREZ, F. El tiempo del territorio, los territorios del tiempo. En: NOGUÉ, J. y ROMERO, J. (editores). Las otras geografías. Valencia: Tirant lo Blanch, 2006, p. 235-254.

NOFRE i MATEO, J. Les politiques culturelles et de la jeunesse dans l'aire métropolitaine de Barcelone: essai critique sur la colonisation culturelle et l'homogénéisation sociale. Sud-ouest européen: revue géographique des Pyrénées et du Sud-Ouest, 2009, No 27, p. 83-95.

OGILVIE, A.G. The time element in Geography. Transactions of the I.B.G.,1953, $\mathrm{N}^{\circ}$ 19 , p. 1-11.

PARKES, D. \& THRIFT, N. Times, spaces, and places. A chronogeographic perspective. Chichester: John Wiley \& Sons, 1980.

PIPITONE. U. Ciudades, naciones, regiones. Los espacios institucionales de la modernidad. México DF: Fondo de Cultura Económica, 2003.

PLANHOL, X. \& CLAVAL, P. Géographie historique de la France. París: Fayard, 1988.

POUNDS, N.J.G. Geografía histórica de Europa. Barcelona: Crítica, 2000.

PRED, A. Place, practice and structure: social and spatial transformation in southern Sweden 1750-1850. Cambridge: Cambridge University Press, 1986.

RANDLE, P.H. El tema del tiempo. Cambio y movimiento en el espacio geográfico. Estudios Geográficos, 1965, Vol. XXVI, № 98, p. 21-40.

RANDLE, P.H. Geografía histórica y planeamiento. Buenos Aires: Eudeba, 1966.

ROMBAI, L. Geografia storica dell'Italia. Ambiente, territori, paessaggi. Florencia: Felice Le Monnier, 2003.

ROSENBERG, D. \& GRAFTON, A. Cartographies of Time. Princeton: Princeton Architectural Press, 2009.

SAMARKIN, V.V. Geografía histórica de Europa occidental en la Edad Media. Madrid: Akal, 1976.

SAUER, C.O. The morphology of the landscape. University of California Publications in Geography, Berkeley (California), Vol. 2, No 2, 1925, p. 19-54.

SCHAEFER, F.K. Excepcionalism in Geography: a methodological examination. An- 
nals of Association of American Geographers, 1953, Vol. 43, p. 205-244.

SCHAEFER, F.K. Excepcionalismo en Geografía. Barcelona: Ediciones de la Universidad de Barcelona, 1977.

SUNYER MARTÍN, P. La geografía histórica y las nuevas tendencias en la geografía humana. En: LINDÓN, A. y HIERNAUX, D. (directores). Los giros de la Geografía Humana. Desafíos y horizontes. Rubí (Barcelona)/ México: Anthropos/Universidad Autónoma Metropolitana, Iztapalapa, 2010, p. 143-173.

TAYLOR, P.J. Modernities. A Geohistorical Interpretation. Cambridge: Polity Press, 1999.

THOMPSON, E.P. Tiempo, disciplina de trabajo y capitalismo industrial. En: THOMPSON, E.P. Costumbres en común. Barcelona: Crítica, 1995, p. 394-452.

THRIFT, N. Time and theory in human geography. Progress in Human Geography, 1977, Vol. I, No 1, p. 65-101, Vol. I, No 3, p. 413-457.

TULLA, A.F. El modelo de difusión de T. Hägerstrand. Una aplicación al Pirineo catalán. Documents d'Anàlisi Geogràfica, 1983, $\mathrm{N}^{\circ} 2$, p. 69-160.

VRIES, J. de. La urbanización de Europa 1500-1800. Barcelona: Crítica, 1987.

WALLERSTEIN, I. El moderno sistema mundial. La agricultura capitalista y los orí- genes de la economía-mundo europea en el siglo XVI. Madrid: Siglo XXI, 1979.

WALLERSTEIN, I. El moderno sistema mundial II El mercantilismo y la consolidación de la economía-mundo europea, 1600-1750. Madrid: Siglo XXI, 1984.

WALLERSTEIN, I. El moderno sistema mundial III. La segunda gran expansión de la economía-mundo capitalista, 1730-1850. Madrid: Siglo XXI, 1999.

WHITTLESEY, D. The horizon of geography. Annals of Association of American Geographers, 1945, № 35, p. 1-36.

WHYTE, I.D. Landscape and History since 1500. Londres: Reaktion Books, 2002.

WOLF, E.R. Europa y la gente sin historia. México DF: Fondo de Cultura Económica, 1987.

ZELINSKY, W. The hypothesis of the mobility transition. Geographical Review, 1971, Vol. 61, No 2, p. 219-249 (la versión utilizada es la traducción al catalán de Montserrat Cuxart y publicada en Treballs de la Societat Catalana de Geografia, № 44, 1997, p. 143173).

ZUSMAN, P. Geografías históricas y fronteras. En: HIERNAUX, D. y LINDÓN, A. (directores). Tratado de Geografía Humana. Rubí (Barcelona)/México: Anthropos/Universidad Autónoma Metropolitana, Iztapalapa, 2006, p. 170-186. 